\title{
Discovering spatiotemporal concepts in discourse
}

Tenbrink, Thora

\section{The Cambridge Handbook of Cognitive Linguistics}

Published: 01/06/2017

Peer reviewed version

Cyswllt i'r cyhoeddiad / Link to publication

Dyfyniad o'r fersiwn a gyhoeddwyd / Citation for published version (APA):

Tenbrink, T. (2017). Discovering spatiotemporal concepts in discourse. In B. Dancygier (Ed.), The Cambridge Handbook of Cognitive Linguistics (pp. 669-683). (Cambridge Handbooks in Language and Linguistics). Cambridge University Press.

\footnotetext{
Hawliau Cyffredinol / General rights

Copyright and moral rights for the publications made accessible in the public portal are retained by the authors and/or other copyright owners and it is a condition of accessing publications that users recognise and abide by the legal requirements associated with these rights.

- Users may download and print one copy of any publication from the public portal for the purpose of private study or research.

- You may not further distribute the material or use it for any profit-making activity or commercial gain

- You may freely distribute the URL identifying the publication in the public portal ?
}

Take down policy

If you believe that this document breaches copyright please contact us providing details, and we will remove access to the work immediately and investigate your claim. 


\section{Chapter 4: Discovering spatiotemporal concepts in discourse.}

Thora Tenbrink, Bangor University, UK

\subsection{Introduction.}

How do we think about space and time, and how is this related to different contexts and situations? To what extent are our thoughts represented in language - and what can we learn from what people say about their current mindset? This chapter addresses these questions by first reviewing findings on conceptual features reflected in spatial and temporal language, and then showing how these can be discovered and analyzed in natural discourse. Speakers' linguistic choices in discourse reflect their underlying concepts in the given situation, and this enables the analyst to gain insights about relevant cognitive aspects through the analysis of language use.

Cognitive Linguistics research has produced a wide range of insights on how the linguistic system (across languages) reveals human concepts of space and time. These ubiquitous domains intricately interrelated, and restricted to a limited range of conceptual patterns that have been subject to diversified analysis. Crucially for the focus of this chapter, both space and time are commonly represented throughout much of natural discourse, as speakers incorporate relevant aspects about these domains in much of their language use. Spatial relationships between people, objects, and locations are regularly conveyed through language, events are linguistically anchored in space and time, different times and events are represented in their relation to each other, and so on. 
Since there is no simple one-to-one mapping between concepts and linguistic expression, speakers need to choose from the available linguistic repertory to convey the concepts that are relevant in a particular discourse context. Languages differ in the linguistic patterns of space and time vocabulary (Sweetser \& Gaby, this volume), and also in the ways in which both domains interact and give rise to conceptual and metaphorical structures (Gijssels \& Casasanto, this volume), resulting in a specific (more or less extensive) repertory of spatiotemporal expressions. Discourse situations affect which of the available options is best suited for current purposes. Referring to an object by its location, as in it's the one over there, for instance, is typically simpler than describing where an object is, as in it is on the right-hand side of the table (Vorwerg \& Tenbrink, 2007). The choice of different formulations for each of these discourse goals (out of the same general repertory) reveals the speakers' different ways of conceptualizing the same scene for communication purposes.

In spite of the fact that space and time are pervasively represented in natural discourse, speakers frequently leave central aspects of a spatiotemporal situation underspecified. Typically, the implied aspects can be inferred from the context; this works in fundamentally different ways for each of the two domains (Tenbrink, 2007b). While temporal language use is often associated with notions of sequentiality and causality, spatial language use implies underlying conceptual reference frames that involve a relatum and a perspective, both of which are often not made explicit. These issues will be explored in more detail below.

Whenever speakers express concepts of time and space in natural discourse, they reveal which aspects of the domains are relevant to them in a particular context 
(Tenbrink, 2012). Patterns of usage reflecting significant concepts can be detected using systematic analysis methods such as those offered by Cognitive Discourse Analysis (CODA; Tenbrink, 2015), which uses controlled empirical settings with experimental variation to elicit systematic differences in conceptualizations as reflected in discourse. This chapter reviews insights gained on spatiotemporal discourse in English by drawing on exploratory as well as controlled empirical studies in the past two decades, introduces CODA as a method, and demonstrates ways in which spatiotemporal concepts can be discovered in discourse.

\subsection{Spatial language in English discourse.}

In many cultures including English-speaking ones, everyday discourse is full of spatial expressions. On the one hand, we talk about what is happening at particular places, where things are, where we will go next, and the like. Being somewhere, and interacting with things and people around us, is central to our lives, and this determines our conversations. On the other hand, spatial language is also the basis for conceptual (metaphorical) transfer of the kind that is extensively discussed in part IV in this book, as in expressions like the bad times are behind us. Here we will focus entirely on the literal use of spatial language, and in particular look at the use of terms indicating relative spatial location, such as on, in, to the left, in front, between, and the like. These and various other terms can be used to locate an object (here called locatum) relative to another object (here called relatum). Frequently, such relationships are expressed through spatial prepositions (on, in, along) or adjectives (close), although other syntactic forms are possible, such as nouns (closeness, 
distance) or adverbs (closely), phrases such as to the left, and the occasional conjunction, as in I don't know where I am (Halliday \& Matthiessen, 2014).

As Talmy (2000) aptly noted, spatial locational terms are highly abstract and schematic. For instance, a term like along makes the schematic assumption that the relatum is linear, as in along a path, in contrast to all over which assumes a planar relatum as in all over the table, or throughout which assumes a volumar relatum as in throughout the aquarium. Our use of these terms, in general, therefore indicates how we think about space: not in terms of metric distances and exact positions, but rather in terms of relative location. And in specific situations of usage, our choice of these terms indicates how we conceptualize the situation. For instance, the phrase along the aquarium indicates a cognitive focus on the length of the aquarium, not its volume.

Objects relate to each other in meaningful ways, and this is reflected not only in the schematic nature of spatial terms, but also in their contextual usage. A term like over indicates not only a geometrically vertical relationship but also a functional relationship between locatum and relatum, as in the umbrella over the man (Coventry, Prat-Sala, \& Richards, 2001). With above, in contrast, function plays a far less central role than the geometrical relation. Choosing over rather than above in a discourse context, then, points to relevance of the functional relationship: the umbrella is over, but not above the man would make sense if the umbrella protects the man from the rain even though it is not above him (i.e., the rain comes from a diagonal angle).

With another class of terms that are often referred to as projective, the underlying concepts are rather intricate. These include terms like left, right, in front, behind and 
some other related terms that involve the projection of a spatial direction on a scene. As we saw in the introductory example, using these terms presupposes an underlying perspective, which is not always made explicit. The combination of a perspective with a particular choice of relatum leads to a range of different options of reference systems. To understand the intended use of projective terms, it is necessary to grasp the underlying reference system - even though this is not directly made explicit in English. Levinson (2003) classified the main options for reference systems as intrinsic and relative (plus a third category, absolute, that relates to concepts such as compass directions, which do not involve projective terms). Relative reference systems use three different positions: locatum, relatum, and origin of perspective (typically the observer), as in to the left of the table from my point of view. In intrinsic reference systems, the origin of perspective is identical with the relatum, as in to my left (see Tenbrink 2011 for more options and details).

Like other spatial terms, projective terms are schematic, and do not normally indicate a precise spatial location. Nevertheless, there are certain spatial limitations for using these terms. When a projective term is used, the locatum will be positioned within a region surrounding an axis (left / right / front / back) with respect to the relatum, based on the conceptualization of a reference system (intrinsic / relative). The size of the region depends on contextual factors, but cannot be wider than a half plane. Locatum and relatum are either externally related to each other, or the locatum is inside the relatum. If one of the objects is in motion (or both), interpretation can become complex, but the main basic distinction between intrinsic and relative reference systems remains valid (Tenbrink, 2011). Generally, any oriented object can serve as the origin of both intrinsic and relative reference systems; the orientation can 
come about by (potential) motion (as in a car) or by intrinsic features such as perception (as in living beings). Furthermore, similar effects arise by functional ordering relations such as those induced by a queue (or other kind of sequence).

Projective terms can occur across a wide range of contexts. Besides denoting the location of objects to one another in a simple configuration, they are equally prominent in route descriptions, where a goal location is described via reference to streets and landmarks that can be easily identified in the real world. In such a context, salience and dimensions of buildings are prominent in the choice of landmarks, and spatial relations are often sufficiently hinted at via simple and vague expressions. For instance, in turn right at the church the spatial relationship between the path and the church remains rather vague, but the traveller will typically be able to identify the church and the relevant road easily enough.

In natural discourse, the reference system that is underlying an utterance can typically not be identified directly on the basis of the linguistic form, since there is no one-toone correspondence between forms and reference systems. Moreover, spatial utterances often leave at least the perspective implicit, and frequently also the relatum (Tenbrink, 2007b) - and both of these are necessary to identify the reference system (Tenbrink, 2011). As a result, the same description is often compatible with various possible conceptual reference systems (Tenbrink, Coventry, \& Andonova, 2011). In summary, spatial terms in English are highly interesting linguistic devices that build on a rich conceptual basis whenever they are used. Discovering their distribution in English discourse highlights important aspects of human conceptualization of spatial relationships. 


\subsection{Temporal language in English discourse.}

Similar to space, human concepts of time are implicitly represented in language. The linguistic means available to speakers, as well as the way temporal language is used in natural communication, reflect speakers' underlying conceptions of the relations between events. However, there is one important difference. While spatial information is completely optional in English, it is impossible to use English grammatically without providing some information about time. Temporal features are conveyed by the main verb through tense and aspect, constituting an indispensible part of any grammatical sentence in English. This allows for expressing intricate temporal relations through variations and combinations of tense and aspect; furthermore, temporal information can be implicitly conveyed via clause order (Dowty, 1986; Halliday \& Matthiessen, 2014). Some non-literal constructs of (originally) spatial relationships also convey temporal relationships, such as Christmas is ahead of us (Boroditsky, 2000, Haspelmath, 1997; Moore, 2006). Since these have been extensively discussed elsewhere in cognitive linguistic approaches, we focus here on concepts conveyed by literal uses of temporal language in English.

On the lexical level, the temporal relational terms before and after (used as prepositions in expressions like before Christmas, and as conjunctions combining clauses as in I woke up after having slept for ten hours) are arguably the most explicit terms available in the language for concepts of temporal relationships. Another frequent explicit term is then, which can convey sequentiality of events. While the repertory of prepositions and adjectives that express relationships directly thus 
appears rather limited in comparison to the spatial domain (see above), the English language offers a large variety of conjunctions or adverbials such as meanwhile, until, by the time and so on (Halliday \& Matthiessen, 2014) that serve to indicate temporal extension and overlap, rather than relating two events to each other. These structural differences in the repertory of linguistic expressions for space as opposed to time reflect fundamentally different conceptualizations of these two domains (Tenbrink, 2007b).

Since temporal order, as such, can be communicated simply by clause order and other grammatical means, the question arises why speakers would want to make it explicit by using one of the few lexical markers for temporal relationships (before, after). One possibility is that the conceptualized relationship is more specific than a mere temporal precedence relation. Herweg (1991) suggested that the terms need to be understood relative to a contextually dependent proximal time frame, i.e., the events need to be sufficiently close to each other in time. This would depend on the conceptually relevant level of granularity (Habel et al. 1993). Example 1 will be understood on a different level of granularity than example 2:

(1) After lunch, James continued his work.

(2) After college, Jill started as a teacher.

In example 1, the proximal time frame of after is determined by the contextual cue lunch, which suggests a scale of minutes or, at best, hours. In contrast, in example 2 the inferred proximal time frame involves weeks or months. However, an increased 
level of granularity does not generally imply an increased duration of the involved events; they are merely considered and represented at a coarser scale.

Apart from the proximal time frame, there may also be an association of immediate succession. In such a case, no other events at the same level of granularity are expected to take place between the two events in question (Miller \& Johnson-Laird, 1976; Herweg, 1991). In example 1, this means that James did not engage in any other substantial activity between lunch and work, and in example 2, Jill did not study or work anywhere else before starting as a teacher. However, these implications can be cancelled easily in an appropriate context, as shown in example 3:

(3) After college, Jill started as a teacher, but she had to work as a shop assistant for a couple of years before finding that job.

Another reason for including an explicit temporal term concerns relevance. If the temporal relationship of two known events, or the length of the time span, is in focus as such, it will need to be communicated clearly and explicitly rather than just being implied through clause order or other grammatical devices. Compare the following:

(4) (Mother to son): You know you are not allowed sweets before dinner - only after.

(5) (Mother to son): You know you are only allowed sweets having had dinner.

The concept of conveying a focused temporal relationship is called 'Regulation' by Tenbrink \& Schilder (2003), and it typically cancels the implication of immediate succession. In example 4, sweets presumably aren't allowed within a reasonable 
amount of time before dinner, but will be allowed after dinner, regardless of other events happening in between. With this concept, the temporal term is often stressed in spoken language (as indicated here by italics).

Further much-discussed aspects of temporal relational terms concern the association with presuppositional effects of different kinds (e.g., Miller \& Johnson-Laird, 1976; Lascarides \& Oberlander, 1993; Tenbrink, 2007a). In example 6, the temporal clause is presupposed, i.e., assumed as known from the discourse context, and it also remains factual if the main clause is negated, as shown in example 7.

(6) Do you need a meal before you hit the road?

(7) No, I don't need a meal before I hit the road.

Moreover, it has frequently been suggested that explicit temporal terms typically carry additional meanings conveying a sense of connection beyond the temporal relationship itself (e.g., Heinämäki, 1974, Schilder, 2001). To gain a sense of this, consider the following utterances:

(8) He talked to her. She decided to leave the school.

(9) After he talked to her, she decided to leave the school.

(10) He talked to her before she decided to leave the school.

The fact that the two events happen in sequential order can be inferred in example 8 through the order of narrating, although other interpretations are possible depending on context. Example 9, however, appears to suggest not only that the events happened 
sequentially, but also that there might be a causal relation between them: She decided to leave the school because he talked to her. Example 10 seems to be less clear in this respect. One possible interpretation is that he attempted to talk her out of deciding to leave the school, which would be a kind of reverse causality. Similarly, a so-called non-veridical reading (Heinämäki, 1974; Miller \& Johnson-Laird, 1976) is conveyed in example 11:

(11) The bomb exploded before hitting the target.

(12) The bomb hit the target after exploding.

In example 11 the inference is clear that the bomb did not hit the target - at least not in the expected way. Example 12 demonstrates that this reading is not available with after: despite common sense, the only available interpretation is that the bomb somehow still managed to hit the target after exploding. Another kind of reverse causality suggests itself in example 13, called 'termination' by Schilder (2001) because the event in the temporal clause terminates the event in the main clause:

(13) Peter waited for Sue for hours before she finally arrived.

As we have seen, the association of causal and other conceptual relationships seems to differ between the temporal relational terms, and it also seems to be a matter of degree rather than a directly licensed inference. Tenbrink \& Schilder (2003) systematically identified the types of non-temporal information conveyed by before, after, and then in a corpus of natural discourse. According to their findings, before and after normally convey additional meanings beyond the temporal relationship, 
while then mostly conveys a sense of immediate succession - much more strongly so than previously suggested for before and after. The implied discourse relations between the clauses connected by the temporal terms are highly context dependent, although they can typically be readily inferred, as illustrated by the examples given so far.

Many of the commonly inferred interpretations are causal in some sense. This fact reflects how humans think of time: not as an abstract domain of unrelated time points, but rather as a network of causally interrelated events (Carston, 2002). Accordingly, it is only natural that our linguistic representations of temporal relations are closely related to notions of causality. However, why would speakers wish to use temporal terms to represent causality? In some situations, some amount of freedom of interpretation may be desirable. In example 9 above, the implication that she decided to leave the school because of his talking to her might not be an 'official' concept that can be communicated explicitly. Alternatively, speakers might be uncertain about this aspect and wish to leave it open so as not to be held responsible. In such cases, it is convenient to use a formulation that allows for more than one interpretation while still getting the main message concerning temporal order across clearly.

Tenbrink (2007b) noted that such conceptual implications remain unparalleled in the spatial domain. While the conceptualized relationship between events often remains implicit in the temporal domain, spatial concepts are normally communicated explicitly. However, unlike the events involved in a verbalized temporal relation, spatial descriptions can leave some of the participants of a spatial relationship implicit, as in the book on the right: this would correspond to an incomplete temporal 
description such as the event before, which is rather rare in English. Moreover, the underlying conceptual reference system can only be inferred when all participants of the spatial relationship are known (locatum, relatum, origin). In this respect, both temporal and spatial descriptions leave important conceptual aspects implicit, but in very different ways.

\subsection{Cognitive Discourse Analysis.}

After having outlined some of the concepts that are conveyed in English discourse representing spatial and temporal relationships, we will now consider how their occurrence can be examined in natural language data. Cognitive Discourse Analysis (CODA, Tenbrink, 2015) offers a systematic methodology for this purpose. The main tenet in CODA is that language offers a broad repertory of options from which speakers choose according to what they perceive as relevant and suitable in a discourse context. Therefore, identifying patterns in speakers' verbalizations in a specific context reveals patterns in their thought and concepts that are relevant in this context. This becomes especially clear through contrast and comparison: Changing the discourse context will change the patterns and features of verbalization in systematic ways.

This idea is related to previous research in various areas. Much psycholinguistic experimentation, for instance, aims at identifying which of two (or more) available options are chosen under what kinds of circumstances. This kind of research has led to the identification of functional relationships underlying the use of spatial terms such as over (see above; Coventry et al., 2001), as well as cross-linguistic and cross- 
cultural differences between uses of reference systems (Levinson, 2003). Further, many cognitive linguists investigate structures in language that reflect structures in the mind (e.g., Evans, 2009), variously focusing on semantics, grammar, metaphorical transfer processes, or other aspects relevant for the relationship between language and thought. What these diverse approaches have in common is the aim of identifying general principles underlying language production or structure. In contrast, CODA aims at highlighting the specific significance of a speaker's choice in a particular situation in which thought plays a distinct role, showing how general cognitive principles are at work in natural discourse, relative to the situational demands.

To achieve this goal, tasks designed to elicit data for CODA allow for unrestricted linguistic choices, aiming for language production in a situation that is as natural as possible - but still controlled with respect to the features of the discourse task, such that changes can be introduced keeping other factors constant. The language data elicited from speakers in different conditions then exhibit patterns that systematically reflect conceptual differences that are relevant in the given context. Unlike psycholinguistic studies, which are typically more narrowly designed, in CODAbased studies it is generally not the case that specific linguistic choices can be predicted. The researcher elicits discourse in natural (but controlled) situations that are cognitively challenging or otherwise relevant to a research question, and examines the data with respect to conceptually meaningful distinctions and recurring patterns across speakers. In this respect, CODA resembles more discursively oriented analysis methodologies such as Conversation Analysis or Critical Discourse Analysis, where language data are approached with an open mind using a systematic analysis procedure. Similar to these methods, CODA aims at identifying particular features of 
discourse; in CODA the specific aim is to discover patterns of language that are particularly revealing concerning the ways in which speakers conceptualize a specific, cognitively interesting or challenging situation. Combinations with other modalities or representations of cognitive processes further highlight the kinds of concepts that are decisive for human cognition in the given situation.

Since not all cognitive processes can be put into words, it needs to be clarified what kinds of experimental procedures are suitable for eliciting meaningful verbal data, including the tricky question of what counts as meaningful. Dealing primarily with complex problem solving tasks, Ericsson \& Simon (1984) offered insightful answers to these issues. The analysis procedures suggested in their (and related) work rely mainly on the content of verbal protocols, focusing on verbalizations of cognitive processes that the speakers are aware of. The content-based inspection of verbal reports, particularly if carried out by experts in the problem domain and set against a substantial theoretical background (Krippendorff, 2004), often leads to well-founded specific hypotheses about the cognitive processes involved. This, in brief, is the state of the art in verbal protocol analysis in cognitive science research. These procedures have been applied in a broad range of areas by researchers across many disciplines concerned with the human mind. However, a close linguistic analysis as suggested in CODA is rarely attempted in this kind of research. The next section will spell out how CODA works in practice for the analysis of spatial and temporal concepts in discourse.

\subsection{Discovering spatial and temporal concepts in English discourse.}


The first step for any CODA approach is to identify a research question with a scope that can be meaningfully addressed by the analysis of language use (Tenbrink, 2015). Generally, CODA is suitable to address questions that pertain to mental representation (the conceptualization of complex scenes, event perception, and the like), and complex cognitive processes (such as problem solving or decision making). Although spatial and temporal concepts are nearly ubiquitously represented in natural discourse (at least in English), not all types of language data are suitable for systematic linguistic analysis if no clear relationship to cognitive effects can be established. Research questions suitable for CODA typically aim at discovering particular types of patterns of spatiotemporal concepts that presuppose a certain kind of context in which they become relevant. The most promising approach is to elicit language in a controlled situation where spatial and/or temporal cognition plays a distinct role.

In the domain of space, as shown above, speakers frequently leave basic elements of a spatial description implicit. To understand the intended meaning, the analyst needs access to the spatial situation in which the language was produced. Out of context, the reference system underlying a description like the box on the left cannot be identified, since it does not contain information about the perspective or the relatum. Likewise, the functional element of over cannot be detected without taking the actual spatial situation into account. In the domain of time, this is somewhat different since the temporal entities (events or times) represented in temporal language (or grammar) are typically not directly perceptually accessible. As a result, most of the information needed for interpretation is often present within the discourse itself, and speakers talk about temporal relationships in a different way than they talk about object relations 
(Tenbrink, 2007b). Nevertheless, to address specific aspects about human concepts of time relative to a specific situation, it will typically be necessary to elicit data in a controlled way, so as to keep the situation and discourse context constant across speakers and identify patterns of usage that reveal patterns of thinking about time (or space). For instance, analyzing descriptions of (or dialogues about) perceived scenes and events reveals the speakers' conceptualizations of spatial and temporal relationships within these scenes and events.

After identifying a suitable research question and scope, the next important step is to clarify the precise way in which data should be collected (Tenbrink, 2015), since different kinds of linguistic data, such as verbal protocols, dialogue data, descriptions, or instructions can reveal cognitive aspects in fundamentally different ways. It is therefore worth examining which linguistic data source would most likely lead to most insights of relevance to the research question. Different conditions allow for establishing relevant contrasts, and can be enhanced by collecting more than one type of linguistic data. Note that CODA, in principle, can also be used to analyze existing language data, such as corpus data (e.g., Danino, 2014; Egorova, Tenbrink, \& Purves, 2015); in such a case, data collection means identifying a suitable data set that can be meaningfully analyzed in this way.

Relevant research in the domain of space has used situations in which participants were asked to describe object configurations of varying complexity (Ehrich \& Koster, 1983; Tenbrink et al., 2011) or maps (Taylor \& Tversky, 1996), to communicate object arrangements in dialogue (Schober, 1995), to describe routes under various circumstances (Denis, 1997; Hölscher, Tenbrink, \& Wiener, 2011), to classify 
direction concepts (Mast et al., 2014), to provide a retrospective report of tour planning (Tenbrink \& Seifert, 2011), and more. In these studies, unrestricted language data were elicited in controlled settings.

To exemplify, Tenbrink et al. (2011) addressed the main strategies speakers use for describing complex object arrangements. We speculated that strategies might differ depending on the extent to which objects were related to each other in a meaningful way. If functional object relationships mattered for spatial description, speakers would refer to a table with chairs around it in a different way than to a shower with nearby chairs. We furthermore aimed to identify the kinds of reference systems that speakers used in this situation. Previous research had mainly used far simpler configurations, leading to contradictory speculations about reference system preferences in English speaking cultures. For instance, while Miller \& Johnson-Laird (1976) proposed that intrinsic reference systems might generally be predominant, Levinson (2003) suggested that in Western cultures, relative reference systems are preferred.

To address these questions, Tenbrink et al. (2011) used a controlled monological spoken discourse task. Participants were asked to describe an arrangement consisting of 15 objects in such a way that a listener would be able to reconstruct the arrangement. In two main conditions, arrangements differed with respect to the availability of functionally meaningful object clusters. The elicited linguistic descriptions were recorded, transcribed, and segmented for purposes of systematic and thorough annotation. 
Following data collection, Tenbrink (2015) recommends a thorough content analysis as speakers may directly express concepts in language that are relevant to the research question. Content analysis can also lead to the identification of conceptual categories to pursue further, based on a closer linguistic analysis. Tenbrink et al. (2011) established through content analysis whether utterances contained reference to the location or the orientation of an object, none of these, or both. This analysis yielded interesting patterns as to the cognitive relevance of object orientation relative to object location. Particularly when orientation seemed to be self-evident in functional object clusters, this information tended to be omitted from description.

The most central task step in CODA is linguistic feature analysis (Tenbrink, 2015). Typically, this comes with a qualitative and a quantitative aspect. Central linguistic features are detected and analyzed with respect to their (known) relation to cognition, drawing on insights in cognitive linguistics such as those represented throughout this book (parts III,IV, and other chapters in the current part VI), functional grammar (Halliday \& Matthiessen, 2014), psycholinguistics (Ellis, 1985-1987), and other resources that highlight the deeper meaning and cognitive significance of particular linguistic features. Systematic annotation then allows for an assessment of the significance of these features within the overall data set, identifying where the relevant features occur, and what characterizes their occurrence. Importantly, any type of data annotation must be reliable, i.e., done in such a way that a different observer will come to the same conclusions. This can be ensured, on the one hand, by a suitable operationalization procedure while annotating, and on the other hand, by using one of various available inter-coder reliability test procedures (e.g., Krippendorff's Alpha as described in Hayes \& Krippendorff, 2007). 
In Tenbrink et al. (2011), the main aim of systematic annotation concerned the identification of spatial reference frames. However, data inspection revealed frequent cases where speakers left the relatum or origin implicit, or both. For this reason, a thorough identification of all underlying reference systems turned out to be impossible - which may serve to explain why the frequently asked question of general reference system preferences is still not resolved. In order to meaningfully address the research question, each individual object description in the collected corpus was annotated with respect to the mention and identity of the relatum, as well as with respect to compatibility with the actual observer's perspective on the scene. It was hypothesized that if speakers departed from this default perspective by using a cognitively effortful mental rotation, this would be related to conceptually meaningful aspects of the configuration (since no different addressee perspective was available that speakers might have used instead). Following systematic annotation, inter-coder reliability was established through applying Krippendorff's Alpha.

The next step following annotation concerns the identification of patterns of distribution of the annotated features within the collected data, for example relative to conditions or at certain decisive moments during the task process (e.g., beginning or end, along with an insight or strategy change, etc.). These patterns are the main results to be discussed and interpreted in light of an in-depth qualitative analysis of the linguistic features identified as crucial in this context, relative to the research question (Tenbrink, 2015). In Tenbrink et al. (2011), results showed that speakers systematically preferred using either the environment as a whole (and its features) as a relatum, or other objects mentioned in directly preceding utterances (independent of 
condition). They normally used their actual (outside) perspective on the scene, except in specific situations where objects were functionally oriented toward each other, or otherwise oriented in a particular way that suggested a different perspective. For instance, one cupboard placed at the left hand wall was frequently used as a relatum for another object next to it on the wall, using the terms left and right. This suggests that the observer imagined being inside the scene facing the cupboard, rather than using an outside perspective, in which the same objects would have been described as being in front of or behind the cupboard. Altogether, the analysis supported the conclusion that relative reference frames dominated over intrinsic ones in our setting, while at the same time suggesting that specific object configuration could induce other types of reference frames. This calls the assumption into question that a general, situation-independent preference for a reference frame can be identified for a culture.

The analysis of linguistic data can often be meaningfully combined with other types of data, such as behavioral performance data, reaction times, and the like. In this final step, the CODA results are related to such other data, or possible extensions such as formalizations or modeling procedures are initiated (Tenbrink, 2015). For the study reported in Tenbrink et al. (2011), a straightforward extension would have been to present another set of participants with the descriptions in order to establish the communicative success of specific strategies. This was, however, left for future investigation.

In the temporal domain, research has mainly focused on the discussion of conceptual phenomena found in discourse examples, rather than eliciting natural language data to examine temporal concepts. This includes research on discourse relations including 
the phenomena discussed above (Lascarides \& Oberlander, 1993; Tenbrink, 2007a; Tenbrink \& Schilder, 2003), space-to-time mappings (Boroditsky, 2000; Haspelmath, 1997; Moore, 2006), temporal reference frames (Chilton, 2013; Tenbrink, 2011), and more. Typically in research of this kind, discourse examples are used to explore concepts and phenomena underlying language use in general. Closer to CODA, research involving elicited data has addressed, for instance, the acquisition of temporal structuring devices in second language learners (Bardovi-Harlig, 2000), use of tense and modality in think-aloud protocols at different stages of a problem solving process (Gralla, 2013), and use of discourse markers indicating the temporal development of a problem solving process (Caron-Pargue \& Caron, 1991; Tenbrink \& Wiener, 2009). Further relevant work addressed the ways in which events are structured and segmented in narratives, in relation to cognitive aspects (Zacks, Tversky, \& Iyer, 2001) and in bilinguals (Bylund, 2011).

However, to my knowledge the issues explored above concerning non-temporal conceptual aspects conveyed by temporal terms have not been addressed using specifically elicited natural language data. A number of predictions emerge from example-based findings in this area, which could be empirically tested. For instance, if (as hypothesized) temporal connectives are preferably used in situations where a causal connection is plausible but not evident, this should lead to systematic patterns in usage (depending on context) that reflect the extent to which a causal relationship is conceptualized by the speaker. Likewise, if after and before differ with respect to presuppositional patterns and associated conceptual relationships, their distribution in natural discourse should differ in ways going far beyond the abstract distinction with 
respect to temporal order, reflecting their distinct roles in representing temporal cognition.

Further promising avenues of research concern the interplay of spatial and temporal concepts in natural discourse, as most empirical work in this area so far has focused on either one of the domains rather than explicitly targeting their interrelations. Tenbrink (2007b) pointed to a range of parallels and contrasts between general principles of language use in the spatial vs. temporal domain, which would lend themselves to further empirical exploration. For instance, as exemplified above, each of the domains leaves central aspects underspecified in spatiotemporal discourse, namely the conceptualized relationship between the entities (events) in the temporal domain, as opposed to the entities themselves (relatum and origin) in the spatial domain. These effects are related to the different discourse functions played by spatial vs. temporal terms that express relations between entities in each domain, and can ultimately be traced back to the fundamental differences in the linguistic repertory for each domain (in English). These effects should result in systematically different usage patterns and conceptualization effects in discourse where both types of relationships are equally relevant, or relevant in different ways. In the vast literature on route directions, both spatial and temporal concepts play a role to some extent, but the main focus is on spatial relationships. Arguably, this reflects the route givers' conceptual focus on the spatial rather than the temporal domain in a motion setting that involves both. It would be interesting to explore scenarios in which the focus of attention shifts more flexibly between space and time, revealing different patterns of discourse to represent each domain. 


\subsection{Conclusion.}

In this chapter, I have reviewed some ways in which spatial and temporal terms in their natural occurrence in discourse convey how humans conceptualize (and, as a result, verbalize) the domains of space and time. To discover the significance of their use in a specific situation context, it is useful to adopt a systematic approach to data collection and analysis from a cognitive perspective, as done in Cognitive Discourse Analysis (Tenbrink, 2015). Research using this and related methods extends traditional discourse and content analysis methods by integrating cognitive linguistic findings about the conceptual significance of specific linguistic patterns. This leads to insights about human thought processes (as reflected in discourse) that the speakers themselves may not be consciously aware of, such as the perspective underlying a spatial description, or the non-temporal concepts conveyed when using temporal terms.

\section{References}

Bardovi-Harlig, Kathleen. 2000. Tense and aspect in second language acquisition: Form, meaning and use. Oxford: Blackwell.

Boroditsky, Lera. 2000. Metaphoric structuring: understanding time through spatial metaphors. Cognition 75, 1-28.

Bylund, Emanuel. 2011. Segmentation and temporal structuring of events in early Spanish-Swedish bilinguals. International Journal of Bilingualism 15:1, 56-84. Caron-Pargue, Josiane and Jean Caron. 1991. Psychopragmatics vs. socioprag-matics: the function of pragmatic markers in thinking-aloud protocols. In Jef Verschueren 
(ed.), Pragmatics at issue: Selected papers of the International Pragmatics

Conference, Antwerp, August 17-22, 1987, Volume I, 29-36. Amsterdam: Benjamins. Carston, Robyn. 2002. Thoughts and Utterances: The pragmatics of explicit communication. Oxford: Blackwell.

Chilton, Paul. 2013. Frames of reference and the linguistic conceptualization of time: present and future. In K.M. Jaszczolt and L. de Saussure (Eds.), Time: Language, Cognition, and Reality. Oxford: Oxford University Press.

Coventry, Kenny R., Merce Prat-Sala, and Lynn V Richards. 2001. The interplay between geometry and function in the comprehension of 'over', 'under', 'above' and 'below'. Journal of Memory and Language 44, 376-398.

Danino, Charlotte. 2014. Language production and meaning construction mechanisms in the discourse on an ongoing event: the case study of CNN's live broadcast on 9/11. PhD Dissertation, University of Poitiers, France.

Denis, Michel. 1997. The description of routes: A cognitive approach to the production of spatial discourse. Cahiers de Psychologie Cognitive, 16(4):409-458. Dowty, David R. 1986. The effect of aspectual class on the temporal structure of discourse: Semantics or Pragmatics? Linguistics and Philosophy 1 (9), 37-62. Egorova, Ekaterina, Thora Tenbrink, and Ross Purves. 2015. Where snow is a landmark: Route direction elements in alpine contexts. Spatial Information Theory: 12th International Conference (COSIT 2015), Santa Fe, October 12-16, 2015. Berlin: Springer.

Ehrich, Veronika and Charlotte Koster. 1983. Discourse Organization and Sentence Form: The Structure of Room Descriptions in Dutch. Discourse Processes 6:169-195. Ellis, A.W. 1985 -1987. Progress in the Psychology of Language, 3 vols. London: Lawrence Erlbaum. 
Ericsson, K. Anders, and Herbert A. Simon. 1984. Protocol analysis-Verbal reports as data. Cambridge, MA: Bradford books/MIT Press.

Evans, Vyvyan. 2009. How words mean: Lexical concepts, cognitive models and meaning construction. Oxford: Oxford University Press.

Gralla, Linn. 2013. Linguistic representation of problem solving processes in unaided object assembly. $\mathrm{PhD}$ dissertation, University of Bremen, Germany.

Habel, Christopher, Michael Herweg, and Simone Pribbenow. 1993. Wissen über Raum und Zeit [Knowledge about space and time]. In: Günther Görz (Ed.), Einführung in die künstliche Intelligenz, 139-204. Bonn: Addison-Wesley. Halliday, Michael A.K. and Christian M.I.M. Matthiessen. 2014. Halliday's Introduction to Functional Grammar (4th edition). London: Routledge.

Haspelmath, Martin. 1997. From Space to Time. Temporal Adverbials in the World's Languages. München: Lincom.

Hayes, Andrew F. and Klaus Krippendorff. 2007. Answering the call for a standard reliability measure for coding data. Communication Methods and Measures, 1, 77-89. Heinämäki, Orvokki. 1974. Semantics of English Temporal Connectives. Dissertation, Dept. of Linguistics, Univ. of Texas, Austin.

Herweg, Michael. 1991. "Temporale Konjunktionen und Aspekt. Der sprachliche Ausdruck von Zeitrelationen zwischen Situationen." Kognitionswissenschaft 2: 51-90. Hölscher, Christoph, Thora Tenbrink, and Jan Wiener. 2011. Would you follow your own route description? Cognition 121, 228-247.

Krippendorff, Klaus. 2004. Content Analysis: An Introduction to its Methodology (2 $^{\text {nd }}$ ed.). London: Sage.

Lascarides, Alex and Jon Oberlander. 1993. Temporal Coherence and Defeasible Knowledge. Theoretical Linguistics 19:1, 1-35. 
Levinson, Stephen C. 2003. Space in Language and Cognition. Cambridge:

Cambridge University Press.

Mast, Vivien, Diedrich Wolter, Alexander Klippel, Jan Oliver Wallgrün, and Thora

Tenbrink. 2014. Boundaries and Prototypes in Categorizing Direction. In Christian

Freksa, Bernhard Nebel, Mary Hegarty, and Thomas Barkowsky (Eds.), Spatial

Cognition 2014, Bremen, Germany, 15-19 September 2014, 92-107.

Miller, George A. and Philip N. Johnson-Laird. 1976. Language and Perception.

Cambridge: Cambridge University Press.

Moore, Kevin E., 2006. Space to time mappings and temporal concepts. Cognitive

Linguistics 17-2, 199-244.

Schilder, Frank. Presupposition triggered by temporal connectives. 2001. In: Miriam

Bras and Laure Vieu (Eds.), Semantic and Pragmatic Issues in Discourse and

Dialogue: Experimenting with Current Dynamic Theories, 85-108. Amsterdam:

Elsevier.

Schober, Michael F. 1995. Speakers, addressees, and frames of reference: Whose effort is minimized in conversations about location? Discourse Processes, 20(2), 219247.

Talmy, Leonard. 2000. Toward a Cognitive Semantics, 2 vols. Cambridge, MA: MIT Press.

Taylor, Holly A. and Barbara Tversky. 1996. Perspective in spatial descriptions. Journal of Memory and Language, 35, 371-391.

Tenbrink, Thora. 2007a. Imposing common ground by using temporal connectives: The pragmatics of before and after. In Anita Fetzer and Kerstin Fischer (Eds.), Lexical Markers of Common Grounds, 113-139. Amsterdam: Elsevier. 
Tenbrink, Thora. 2007b. Space, time, and the use of language: An investigation of relationships. Berlin: Mouton de Gruyter.

Tenbrink, Thora. 2011. Reference frames of space and time in language. Journal of Pragmatics 43:3, 704-722.

Tenbrink, Thora. 2012. Relevance in spatial navigation and communication. In Cyrill Stachniss, Kerstin Schill, and David Uttal (Eds.), Spatial Cognition 2012, 358--377. Springer, Heidelberg.

Tenbrink, Thora. 2015. Cognitive Discourse Analysis: Accessing cognitive representations and processes through language data. Language and Cognition 7:1, 98 $-137$.

Tenbrink, Thora, Kenny R. Coventry, and Elena Andonova. 2011. Spatial strategies in the description of complex configurations. Discourse Processes 48:237-266. Tenbrink, Thora and Frank Schilder. 2003. (Non)temporal concepts conveyed by before, after, and then in dialogue. In: Peter Kühnlein, Hannes Rieser and Henk Zeevat (Eds.): Perspectives on Dialogue in the New Millennium. Amsterdam and Philadelphia: John Benjamins, pp 353-380.

Tenbrink, Thora and Inessa Seifert. 2011. Conceptual layers and strategies in tour planning. Cognitive Processing 12:1, 109-125.

Tenbrink, Thora and Jan Wiener. 2009. The verbalization of multiple strategies in a variant of the traveling salesperson problem. Cognitive Processing 10:2, 143-161. Vorwerg, Constanze and Thora Tenbrink. 2007. Discourse factors influencing spatial descriptions in English and German. In Thomas Barkowsky, Markus Knauff, Gérard Ligozat, and Dan Montello (Eds.), Spatial Cognition V: Reasoning, Action, Interaction, 470-488. Berlin: Springer. 
Zacks, Jeff M., Barbara Tversky, and Gowri Iyer. 2001. Perceiving, remembering, and communicating structure in events. Journal of Experimental Psychology: General, 130, 29-58. 